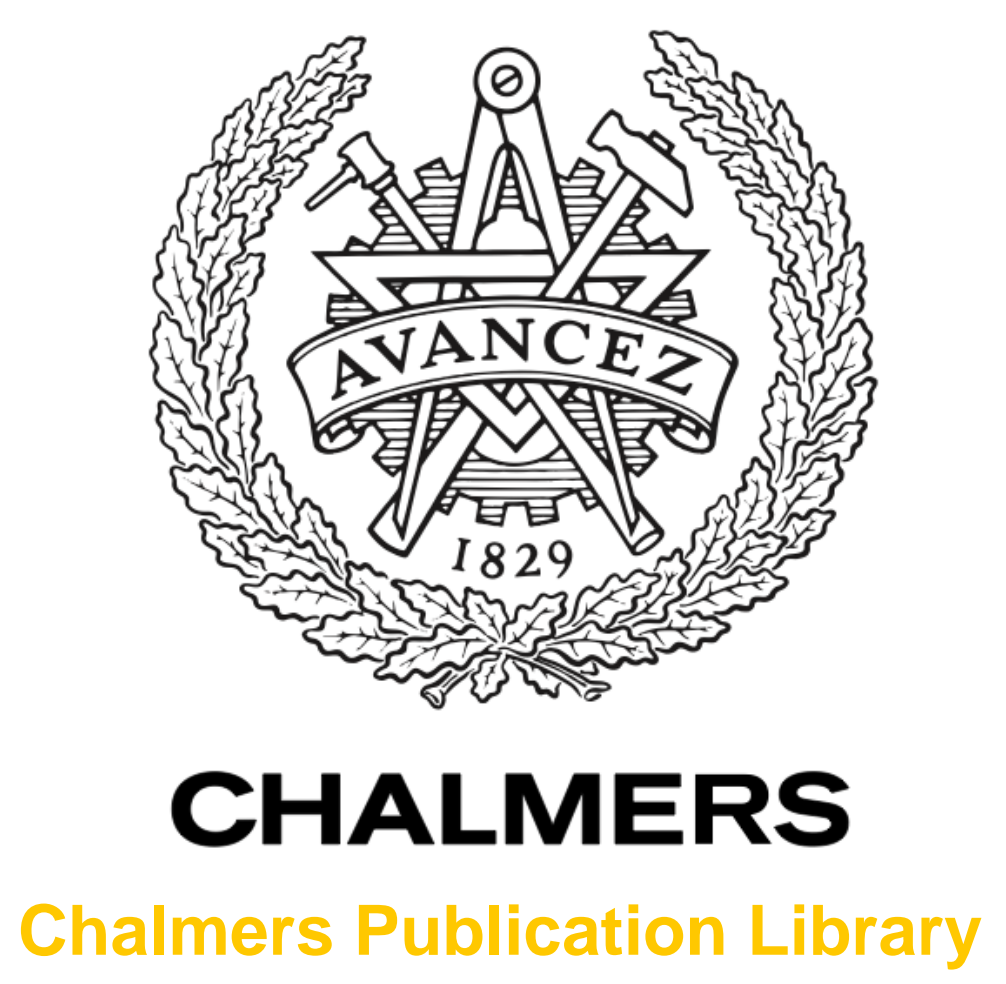

\title{
Second-Generation Fluorescent Quadracyclic Adenine Analogues: Environment- Responsive Probes with Enhanced Brightness
}

This document has been downloaded from Chalmers Publication Library (CPL). It is the author's version of a work that was accepted for publication in:

Chemistry - A European Journal (ISSN: 0947-6539)

\author{
Citation for the published paper: \\ Dumat, B. ; Bood, M. ; Sandberg Wranne, M. et al. (2015) "Second-Generation Fluorescent \\ Quadracyclic Adenine Analogues: Environment-Responsive Probes with Enhanced \\ Brightness". Chemistry - A European Journal, vol. 21(10), pp. 4039-4048.
}

http://dx.doi.org/10.1002/chem.201405759

Downloaded from: http://publications.lib.chalmers.se/publication/215167

Notice: Changes introduced as a result of publishing processes such as copy-editing and formatting may not be reflected in this document. For a definitive version of this work, please refer to the published source. Please note that access to the published version might require a subscription. 


\title{
Second-generation fluorescent quadracyclic adenine analogues: Environment responsive probes with enhanced brightness
}

\author{
Blaise Dumat, ${ }^{[a] \S}$ Mattias Bood, ${ }^{[b] \S}$ Moa S. Wranne, ${ }^{[a]}$ Christopher P. Lawson, ${ }^{[b]}$ Anders Foller Larsen, ${ }^{[a]}$ \\ Søren Preus, ${ }^{[c]}$ Jens Streling, ${ }^{[b]}$ Henrik Gradén, ${ }^{[d]}$ Eric Wellner, ${ }^{[d]}$ Morten Grøtli[b]* and L. Marcus \\ Wilhelmsson ${ }^{[\mathrm{a}] \star}$
}

\begin{abstract}
Fluorescent base analogues comprise a group of increasingly important molecules for the investigation of nucleic acid structure, dynamics and interactions with other molecules. Herein, we report on the quantum chemical calculation-aided design, synthesis and characterization of four new putative quadracyclic adenine analogues. The compounds were efficiently synthesized from a common intermediate via a two-step pathway with the Suzuki-Miyura coupling as the key step. Two of the compounds, qAN1 and qAN4, display brightnesses $\left(\varepsilon \Phi_{\mathrm{F}}\right)$ of 1700 and 2300 respectively, in water and behave as wavelength-ratiometric $\mathrm{pH}$ probes under acidic conditions. The other two, qAN2 and qAN3, display lower brightnesses, but exhibit polarity sensitive dual-band emissions that could prove useful to investigate DNA structural changes induced by DNA-protein or -drug interactions. The four qANs are very promising microenvironment sensitive fluorescent adenine analogues displaying a considerable brightness for such compounds.
\end{abstract}

\section{Introduction}

Fluorescence spectroscopy and microscopy are sensitive and versatile techniques perfectly suited for the investigation of the structure and function of nucleic acids. ${ }^{[1]}$ Fluorescence emission can be modulated by and report on a variety of environmental factors and photophysical events such as polarity, $\mathrm{pH}$, energy transfer and excited-state reactions. In addition to the signal intensity, more complex parameters such as fluorescence lifetime or anisotropy can also be measured and further increase the analytical possibilities offered by fluorescence spectroscopy. ${ }^{[2]}$ Another advantage of fluorescence spectroscopy is its temporal resolution which enables monitoring of dynamic processes on short time scales. The recent

[a] Ms. M. Sandberg (MS), Dr. B. Dumat (BD), Dr. A .F. Larsen (AFL), Prof. L. M. Wilhelmsson (LMW)

Department of Chemical and Biological Engineering/Chemistry and Biochemistry, Chalmers University of Technology, S-41296 Gothenburg, Sweden.

E-mail: marcus.wilhelmsson@chalmers.se.

[b] Mr. M. Bood (MB), Dr. C. P. Lawson (CPL), Mr. J. Streling (JS), Prof. M. Grøtli (MG)

Department of Chemistry, Medicinal Chemistry, University of Gothenburg, S-41296 Gothenburg, Sweden. E-mail: grotli@chem.gu.se.

[c] Dr. S. Preus (SP)

Interdisciplinary Nanoscience Center (iNANO), Aarhus University, Aarhus C, DK-8000, Denmark.

[d] Dr. H. Gradén (HG), Dr. E. Wellner (EW)

AstraZeneca R\&D Mölndal, S-431 83 Mölndal, Sweden.

$\S \quad$ Both authors contributed equally to the work

Supporting information for this article is given via a link at the end of development of single molecule multiparameter fluorescence detection is one example of the new possibilities offered by advanced fluorescence techniques, potentially giving access to quantitative measures of nucleic acid structural conformations and dynamics at the single molecule level. ${ }^{[3]}$

Due to the virtually non-fluorescent character of the natural nucleobases, the study of DNA/RNA by fluorescence spectroscopy requires labeling. ${ }^{[4]}$ One labeling strategy involves tethering external fluorophores onto the DNA sequence by chemical means, to either the nucleobases or the backbone. This method benefits from the wide variety of bright, small organic fluorophores available with a wide range of desirable spectral and/or photophysical properties..$^{[5]}$ However, these probes are usually tethered to the DNA via long flexible linkers that induce considerable mobility resulting in uncertainty regarding the orientation and position of the probes relative to the DNA. Moreover, these bulky probes can significantly perturb the structure of the native DNA and thus disrupt the delicate balance between structure and biological function. For some applications these issues can be overlooked, as in the case of single molecule studies where a high brightness is mandatory and the effects of probe orientation can be partly compensated for, ${ }^{[6]}$ but not for other applications where detailed information about the intrinsic properties of the DNA is required.

A complementary DNA labeling method involves incorporation of synthetic fluorescent analogues as replacements for the natural nucleobases. These so-called fluorescent base analogues (FBAs) are fluorescent molecules capable of closely mimicking the properties of the natural bases and in particular their selective base-pairing ability. ${ }^{[7]} A$ wide range of molecules with often very different characteristics fall under this category, like the size-expanded nucleobases ${ }^{\left[{ }^{[8]}\right.}$ or even fluorescent hydrocarbons with little similarity to the native nucleobases. ${ }^{[9]}$ The latter are thus often referred to as base surrogates rather than base analogues. Due to the need to find the right balance between biochemical and fluorescent properties, FBAs cannot usually compete with the brightness of external fluorophores. However, they are still a highly valuable complementary labeling method. They can be site-specifically incorporated into oligonucleotides by solid-phase synthesis, they are usually less disruptive of the overall DNA architecture than external probes and since they are firmly stacked in the nucleic acids assembly, they report on the intrinsic properties of the DNA/RNA structure rather than on their own dynamics. For extensive reviews on the subject, the reader is referred to the reviews by Wilson et al., Dodd et al., Sinkeldam et al., Wilhelmsson and references cited therein. ${ }^{[10]}$ The utilisation of FBAs was first naturally focused on the study of the numerous bioprocesses in which DNA and RNA are involved, ${ }^{[11]}$ but more recently they have outgrown the field of strict nucleic acid biology and have for instance even proven useful in the 
development of DNA-based optical chemosensors ${ }^{[12]}$ and in DNA nanotechnology. ${ }^{[13]}$

Ever since the first reports on FBAs by Stryer et al. ${ }^{[14]}$ and Leonard and coworkers ${ }^{[15]}$ over four decades ago, considerable efforts have been put into the development of new nucleobase replacements. In spite of the huge number of analogues reported in the literature, ${ }^{[10]}$ their design still remains an ongoing challenge due to the inherent difficulty of satisfying all the design criteria. A typical design strategy is to extend the $\pi$-conjugation of the purine/pyrimidine system to increase the maximum absorption wavelength, the molar absorptivity and thereby presumably also improve the fluorescence properties. This apparently simple approach is rendered more difficult by the necessity to retain the properties of the natural nucleobase at the same time, which means keeping the size of the molecule as close as possible to that of the original structure while retaining the base-pairing ability. Adding to the difficulty, most FBA monomers are often highly quenched once incorporated into DNA or RNA. For example, one of the earliest and most used FBAs, 2-aminopurine (2-AP), has a high quantum yield as a monomer (0.68) which is quenched approximatly 100 -fold inside DNA resulting in a low brightness in nucleic acid measurements. ${ }^{[14]}$

Using the strategy mentioned above, we have previously developed the first FRET pair consisting of tricyclic cytosine analogues that was successfully used for quantitative FRET inside DNA. ${ }^{[16]}$ More recently, we also reported on a series of triazole adenine analogues ${ }^{[17]}$ and on a quadracyclic adenine analogue qA (Figure1A). ${ }^{[18]}$ Pursuant to our continued interest in this area, we report herein on the successful use of quantum chemical calculation-aided design to develop four fluorescent putative quadracyclic adenine analogues (qAN1, qAN2, qAN3 and qAN4, Figure1B). In agreement with the quantum calculations, we find that two of the four compounds display a significantly higher brightness compared to qA. All four compounds are sensitive towards their microenvironment, and are promising for the study of nucleic acids by fluorescence spectroscopy.

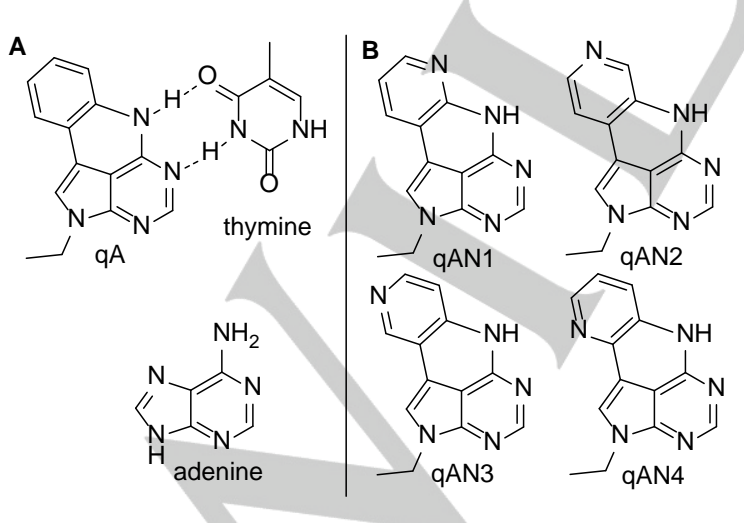

Figure 1. A: Structure of adenine and the quadracyclic adenine analogue qA base-paired with thymine. B: Structure of the new adenine analogues, qAN1, qAN2, qAN3 and qAN4.

\section{Results and Discussion}

\section{Design of new quadracyclic adenine analogues}

The fluorescent nucleobase $\mathrm{qA}$ has been shown to be an excellent adenine analogue, introducing only minimal perturbation to the DNA structure and pairing selectively with thymine ${ }^{[18]}$ In its monomeric form, qA has rather modest optical properties $\left(\varepsilon=5000 \mathrm{M}^{-1} \mathrm{~cm}^{-1}\right.$ at $\left.355 \mathrm{~nm}, \Phi_{\mathrm{F}}=0.068\right)$ and it is additionally quenched, about 10 -fold, inside DNA. The fairly low resulting brightness in DNA $\left(\varepsilon \Phi_{\mathrm{F}} \approx 30\right)$ is nonetheless in the same range as that of $2-\mathrm{AP}\left(\varepsilon \Phi_{\mathrm{F}} \approx 50\right)$ which remains a standard in the field although it has been shown to perturb both DNA structure and dynamics. ${ }^{[19]}$

In an attempt to improve on the photophysical properties of quadracyclic adenine, we set out to vary the outer ring of qA which provides several possibilities for chemical modifications that could potentially improve the optical properties without interfering with the hydrogen-bonding face. Using quantum chemical calculations we estimated the geometries and spectroscopic properties of a set of qA-derivatives where the outer benzene ring is altered to become an electron accepting heterocycle (pyridine or pyrimidine), thus potentially modifying the electronic configuration of the compounds and their spectroscopic properties. TDDFT (Time Dependent Density Functional Theory) calculations were performed for several members of the qA family to help select the target compounds. TDDFT with the B3LYP functional was chosen over more exact wavefunction-based methods due to the fairly large size of the investigated compounds. A CPCM solvation model for $\mathrm{H}_{2} \mathrm{O}$ was also added. The same calculation model and conditions had previously been used with accurate results to predict the electronic transitions of $\mathrm{qA}^{[18]}$ and of the family of tricyclic cytosine analogues. ${ }^{[20]}$ We therefore reused them here to assess the likelihood of our target compounds exhibiting improved properties compared to qA.

The results of the calculations of the three lowest singlet energy transitions (only considering oscillator strengths $>0.001$ ) of the four qAN pyridine derivatives (Figure1B) and their associated oscillator strengths are shown in Table 1 . The corresponding values for $\mathrm{qA}$ are also shown for comparison.

Table 1. The three lowest electronic transitions ${ }^{[a]}$ of the $\mathrm{qAN}$ derivatives as predicted by TDDFT B3LYP/6-311+G(2d) calculations on DFT B3LYP/6$31 \mathrm{G}(\mathrm{d}, \mathrm{p})$ optimised geometries with a CPCM solvation model for $\mathrm{H}_{2} \mathrm{O}$.

\begin{tabular}{cccccccccc}
\hline \multicolumn{2}{c}{$q \mathrm{qA}$} & \multicolumn{2}{c}{$\mathrm{qAN1}$} & \multicolumn{2}{c}{$\mathrm{qAN2}$} & \multicolumn{2}{c}{$\mathrm{qAN3}$} & \multicolumn{2}{c}{$\mathrm{qAN4}$} \\
$\lambda^{[\mathrm{b}]}$ & $\mathrm{f}$ & $\lambda^{[\mathrm{b}]}$ & $\mathrm{f}$ & $\lambda^{[\mathrm{b}]}$ & $\mathrm{f}$ & $\lambda^{[\mathrm{b}]}$ & $\mathrm{f}$ & $\lambda^{[\mathrm{b}]}$ & $\mathrm{f}$ \\
\hline 350 & 0.060 & 359 & 0.20 & 360 & 0.041 & 359 & 0.035 & 355 & 0.30 \\
330 & 0.02 & 341 & 0.083 & 337 & 0.22 & 324 & 0.18 & 347 & 0.003 \\
283 & 0.1 & 281 & 0.091 & 277 & 0.073 & 279 & 0.082 & 283 & 0.10 \\
\hline
\end{tabular}

Overall the results show no major changes in the predicted spectral properties. All four new compounds display a lowest energy transition between 350 and $360 \mathrm{~nm}$ while that of qA is found at $350 \mathrm{~nm}$. However, compared to $\mathrm{qA}$, the oscillator strengths ( $f$ ) of the lowest transition of qAN1 and qAN4 are three to five times higher. Since $f$ in theory is proportional to the molar 
absorptivity, qAN1 and qAN4 can be expected to absorb more strongly in the lower energy part of the spectrum. Importantly, since the oscillator strength of the $S_{0} \rightarrow S_{1}$ transition is, through the Strickler-Berg relation, ${ }^{[21]}$ proportional to the radiative decay rate $\left(\mathrm{k}_{\mathrm{r}}\right), \mathrm{qAN} 1$ and $\mathrm{qAN} 4$ can therefore be expected to be more fluorescent than $\mathrm{qA}$, provided that their respective non-radiative decay rates $\left(k_{n r}\right)$ are similar. On the contrary, the oscillator strengths of the $S_{0} \rightarrow S_{1}$ transition for both qAN2 and qAN3 are lower than that for $q A$ but they display stronger $S_{0} \rightarrow S_{2}$ transitions at $337 \mathrm{~nm}$ and $324 \mathrm{~nm}$, respectively. This predicts an absorption in the $330 \mathrm{~nm}$-region similar to that of $\mathrm{qA}$ and possibly suggests weaker fluorescence properties.

The results from the calculations for the pyrimidine derivatives (Figure S1) suggest a red-shift in the absorption of about 20 to $30 \mathrm{~nm}$ but the oscillator strength value for the $S_{0} \rightarrow S_{1}$ transition is at best 0.31 which is comparable to the values of the most promising qAN derivatives qAN1 and qAN4. From the retrosynthetic analysis it was envisioned that the synthesis of the pyrimidine derivatives would be significantly more challenging than for the pyridine qAN-derivatives. Since the calculations suggested that it would bring no improvement to the optical properties, we therefore decided to synthesize only the qANderivatives. The quadracyclic adenine analogue candidates, qAN1 and qAN4, with improved predicted spectroscopic properties relative to qA were thus chosen as the initial target compounds. In order to obtain sufficient data to perform a small SAR study and test the accuracy of the TDDFT calculations, the series was completed with the less promising derivatives qAN2 and qAN3.

\section{Synthetic Procedures}

The series of qAN analogues were synthesised in 2 steps from a common intermediate $\mathbf{3}$, which itself is readily accessed in 2 synthetic steps beginning from commercially available 6chloro-7-iodo-7-deazapurine (1) (Scheme 1).

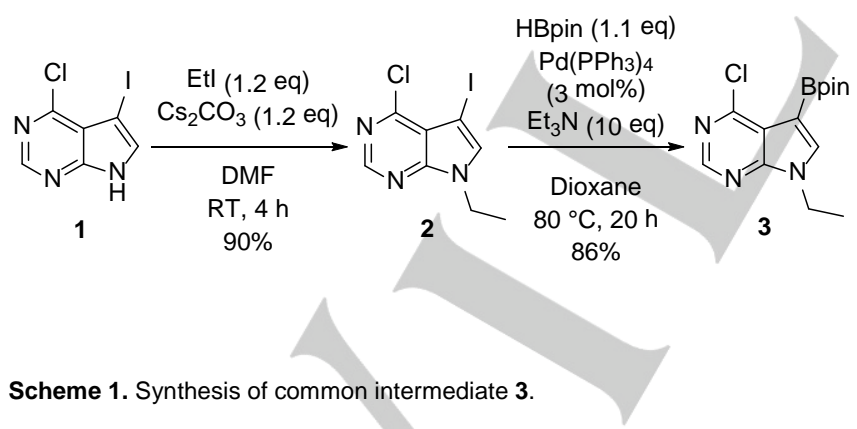

Alkylation utilizing ethyl iodide and cesium carbonate in DMF at room temperature afforded 2 in a very good yield (90\%), isolated by precipitation from water and used without further purification. Miyaura-borylation with pinacolborane in dioxane at $80{ }^{\circ} \mathrm{C}$ for $24 \mathrm{~h}$ with tetrakis(triphenylphosphine)palladium(0) as catalyst afforded 3 in a good yield (86\%), after purification by flash chromatography.
It was envisioned that a cross-coupling reaction followed by cyclisation via a $S_{N} A r$ reaction would be a convenient strategy to rapidly generate the desired series of diverse analogues by simply varying the substitution pattern on the pyridine ring coupling partner (4) (Scheme 2). A catalyst screen was therefore conducted to identify suitable conditions for the reaction, the results of which are summarised below (Table 2 ).

All reactions were based on literature procedures, ${ }^{[22]}$ but run at $80{ }^{\circ} \mathrm{C}$ for 24 hours, after which the conversion was determined by UPLC-MS analysis.

Table 2. Suzuki-Miyaura catalyst screen

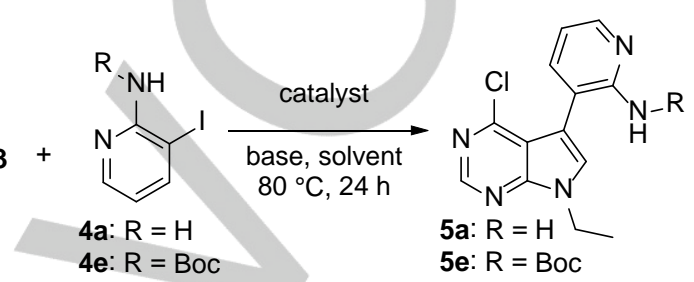

\begin{tabular}{|c|c|c|c|c|c|}
\hline \multirow[t]{2}{*}{ Entry } & \multirow[t]{2}{*}{ Catalyst } & \multirow[t]{2}{*}{ Solvent } & \multirow[t]{2}{*}{ Base } & \multicolumn{2}{|c|}{ Conversion ${ }^{\mathrm{a}]}$} \\
\hline & & & & $5 a[\%]$ & 5e [\%] \\
\hline $1^{[46]}$ & $20 \% \mathrm{Pd}\left(\mathrm{PPh}_{3}\right)_{4}$ & DMA & $\mathrm{Cs}_{2} \mathrm{CO}_{3}$ & 27 & 54 \\
\hline $2^{[47]}$ & $\begin{array}{l}1 \% \mathrm{Pd}_{2}(\mathrm{dba})_{3} \\
2.4 \% \mathrm{PCy}_{3}\end{array}$ & $\begin{array}{l}\text { dioxane- } \\
\mathrm{H}_{2} \mathrm{O} 1: 1\end{array}$ & $\mathrm{~K}_{3} \mathrm{PO}_{4}$ & 16 & 59 \\
\hline $3^{[48]}$ & $\begin{array}{l}2 \% \mathrm{Pd}(\mathrm{OAc})_{2} \\
2.4 \% \text { Xphos }\end{array}$ & $\begin{array}{l}n-\mathrm{BuOH}-\mathrm{H}_{2} \mathrm{O} \\
4: 1\end{array}$ & $\mathrm{CsOH}$ & 16 & n.r. \\
\hline $4^{[49]}$ & $5 \% \mathrm{PdCl}_{2}\left(\mathrm{PPh}_{3}\right)_{2}$ & $\begin{array}{l}\mathrm{MeCN}-\mathrm{H}_{2} \mathrm{O} \\
1: 1\end{array}$ & $\mathrm{~K}_{3} \mathrm{PO}_{4}$ & 75 & 33 \\
\hline $5^{[50]}$ & $3 \% \mathrm{Pd}(\mathrm{dtbpf}) \mathrm{Cl}_{2}$ & $\mathrm{MeCN}$ & $\mathrm{Na}_{2} \mathrm{CO}_{3}$ & 1 & n.r. \\
\hline $6^{[51]}$ & $1 \%\left(\mathrm{Pd}(\mathrm{Br})_{2} \mathrm{PtBu}_{3}\right)_{2}$ & THF- $\mathrm{H}_{2} \mathrm{O} \quad 1: 1$ & $\mathrm{KF}^{[\mathrm{c}]}$ & n.r. & n.r. \\
\hline $7^{[52]}$ & $5 \%$ PEPPSI-IPr & $\mathrm{MeOH}$ & $\mathrm{KF}^{[\mathrm{c}]}$ & 74 & 39 \\
\hline $8^{[50]}$ & $\begin{array}{l}3 \%[\mathrm{Pd}(\mu-\mathrm{Br}) t- \\
\left.\mathrm{Bu}_{3} \mathrm{P}\right]_{2}\end{array}$ & dioxane & $\mathrm{Na}_{2} \mathrm{CO}_{3}$ & n.r. & 58 \\
\hline $9^{[50]}$ & $\begin{array}{l}3 \% \mathrm{PdCl}_{2}(\mathrm{P}(\mathrm{o}- \\
\left.\mathrm{Tol})_{3}\right)_{2}\end{array}$ & dioxane & $\mathrm{K}_{3} \mathrm{PO}_{4}$ & n.r. & n.r. \\
\hline $10^{[53]}$ & $\begin{array}{l}2 \% \mathrm{Pd}_{2}(\mathrm{dba})_{3} \\
4 \% \text { Q-Phos }\end{array}$ & toluene & $\mathrm{K}_{3} \mathrm{PO}_{4}$ & 27 & 14 \\
\hline $11^{[54]}$ & $\begin{array}{l}2 \% \mathrm{Pd}(\mathrm{OAc})_{2} \\
4 \% \text { S-Phos }\end{array}$ & $n-\mathrm{BuOH}$ & $\mathrm{K}_{3} \mathrm{PO}_{4}$ & 24 & 19 \\
\hline $12^{[55]}$ & $\begin{array}{l}5 \% \mathrm{Pd}(\mathrm{OAc})_{2} \\
10 \% \mathrm{dppf} \\
1 \mathrm{eq} \mathrm{CuCl}\end{array}$ & DMF & $\mathrm{Cs}_{2} \mathrm{CO}_{3}$ & 48 & 64 \\
\hline
\end{tabular}
( $40 \%$ on alumina) was used.

To determine the need for protection of the aniline nitrogen, both the free aniline and the $N$-Boc protected aniline were evaluated in the reactions. This highlighted two sets of conditions (Table 2, entries 4 and 7) which afforded similarly high conversions in the test system and proved that protection of the aniline was not required and was in fact detrimental. In spite of the high conversion achieved the conditions used in entry 7 were deemed to be unsuitable for use in future nucleoside synthesis, because KF (40\% on alumina) was used as a base. Attempts to replace KF as a base were unsuccessful, so the use of the PEPPSI system was discontinued. Further investigations around the reaction conditions used in entry 4 revealed that increasing the catalyst loading to $10 \%$ led to extensive protodeborylation of the purine starting material (data not shown). At room temperature, the reaction proceeded much slower with complete consumption of starting materials after 6 hours of reaction time, affording an isolated yield of $61 \%$ (data 
not shown). However, reduction of the catalyst loading to $3 \%$ improved the observed conversion and the isolated yields (Scheme 2). It was also possible to simultaneously reduce the amount of aniline used to 1.1 equivalents, while still maintaining good yields (Scheme 2).

These enhanced reaction conditions were successfully applied to the synthesis of all four qAN analogues utilizing the corresponding anilines to afford the Suzuki-Miyaura crosscoupling products $\mathbf{5 a - d}$ (Scheme 2). The yields of $\mathbf{5 a - c}$ were generally good, but in the case where the pyridine nitrogen was located ortho to the iodine (4d), a decrease in conversion was observed.

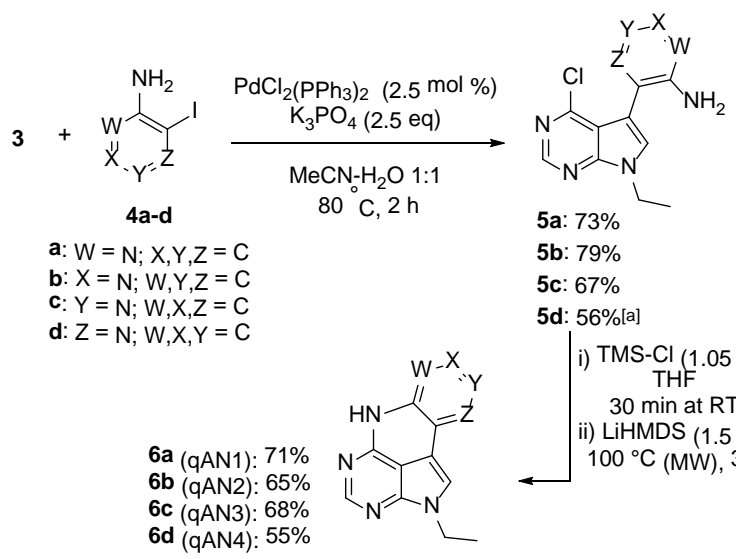
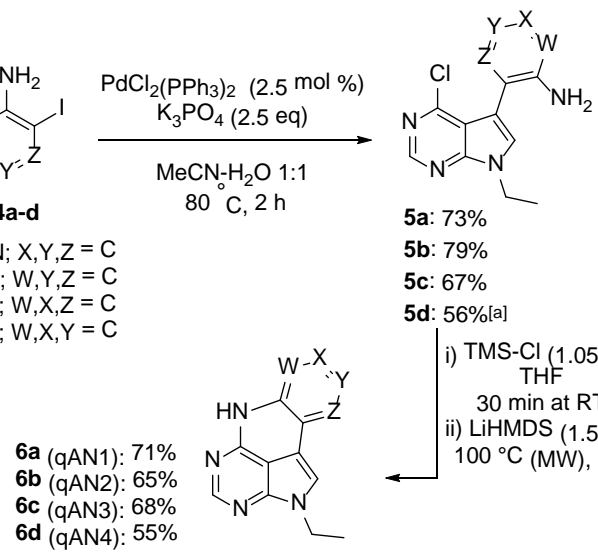

and are summarized in Table 3 . The large aromatic ring systems make the qAN derivatives hydrophobic resulting in a limited solubility in water and measurements start deviating from BeerLambert's law slightly above $10 \mu \mathrm{M}$. The spectral properties of the compounds were also investigated under acidic $\mathrm{pH}$ conditions and in a variety of aprotic and protic solvents covering a wide polarity range.

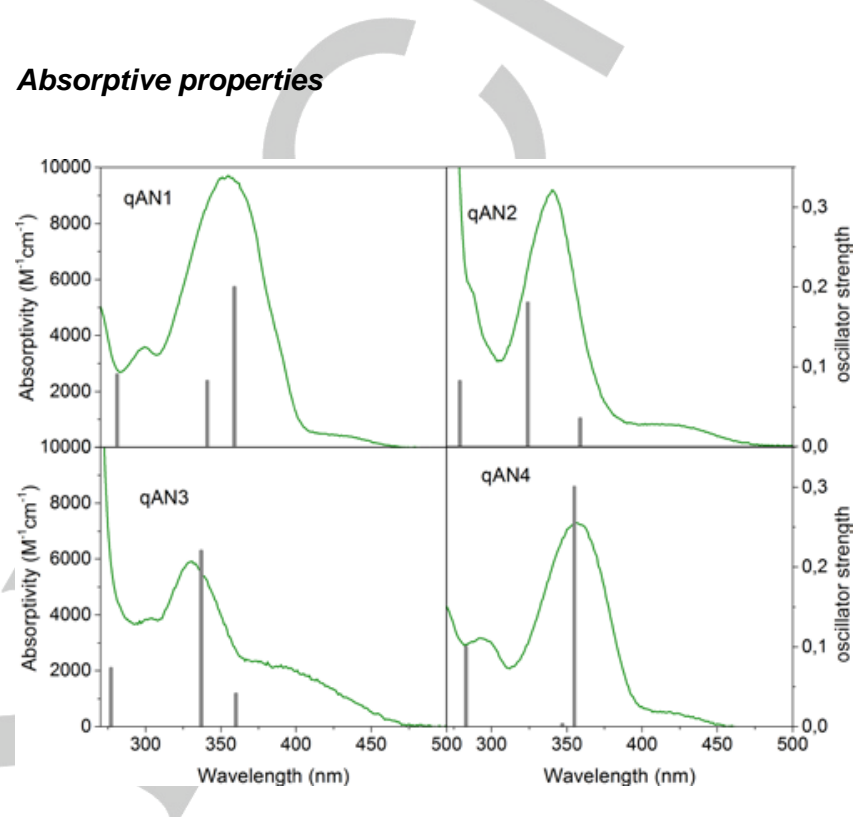

Figure 2. Absorption spectra of the qAN family and corresponding oscillato strengths of the three lowest electronic transitions calculated by TDDFT. Spectra were measured at room temperature in milliQ water. The small shoulders at longer wavelengths are attributed to a residual protonation of the structures.

lower yield of $\mathbf{5 d}$ was attributed to the formation significant amounts of homo-coupled deaza-purine material presumably because the reactivity of $\mathbf{4 d}$ in the cross-coupling reaction is reduced when the pyridine nitrogen is positioned ortho to the iodine. The competing homo-coupling reaction of the borylated purine starting material therefore becomes more predominant, which reduces the final yield of the material obtained. To reduce the rate of activation of $\mathbf{3}$ the reactionsolvent in the case of $\mathbf{4 d}$ was changed to anhydrous DMA, which allowed for the isolation of $\mathbf{5 d}$ in $56 \%$ yield.

The final ring-closing reaction was initially attempted utilizing LiHMDS at $100^{\circ} \mathrm{C}$, which had previously been shown to work well in the synthesis of the $N$-methylated analogue MeqAN1, (See Supporting information), but this resulted in complex mixtures.

It has been reported that the nucleophilicity of nitrogen can be increased by silylation. ${ }^{[23]}$ Pre-stirring of $\mathbf{5 a - d}$ with chlorotrimethylsilane (TMS-Cl), followed by addition of an excess of LiHMDS before heating to $100^{\circ} \mathrm{C}$ for 2 hours afforded the desired products (6a-d) in moderate to good yields across the pyridine series.

\section{Photophysical characterisation}

The steady-state and time-resolved spectroscopic properties of the qAN derivatives were thoroughly characterized in water

As predicted by the quantum chemical calculations, the qAN derivatives absorb approximately in the same range as qA with lowest absorption bands centered on $360 \mathrm{~nm}$ for qAN1 and qAN4 and $335 \mathrm{~nm}$ for qAN2 and qAN3 (Figure 2). Like their parent compound, all qAN derivatives have absorption maxima clearly distinct from the natural bases of DNA allowing for a selective excitation inside nucleic acid systems. In general the molar absorptivities are stronger for the qAN family compared to qA with an increase of up to two-fold (Table 3). The absorptions of qAN1 and qAN4 are red-shifted by approximately $30 \mathrm{~nm}$ compared to $\mathrm{qA}$ and the principal low absorption bands (9500 and $7300 \mathrm{M}^{-1} \mathrm{~cm}^{-1}$, respectively) are found at wavelengths well in line with the energies of the $S_{0} \rightarrow S_{1}$ transitions found in the TDDFT calculations. The absorption maxima of the principal low energy bands for qAN2 and qAN3 lie in the same range and are more intense than for the corresponding peak in qA. They are found between the TDDFT-predicted $S_{0} \rightarrow S_{1}$ and $S_{0} \rightarrow S_{2}$ transitions for qAN2 $\left(9450 \mathrm{M}^{-1} \mathrm{~cm}^{-1}\right)$ and at the predicted $\mathrm{S}_{0} \rightarrow \mathrm{S}_{2}$ transition for qAN3 $\left(5900 \mathrm{~m}^{-1} \mathrm{~cm}^{-1}\right)$. In the case of qAN4, the lowest energy absorption band is predicted to be highly dominated by or even purely composed of the $S_{0} \rightarrow S_{1}$ transition, a fact which will be a major advantage for FRET or anisotropy 
measurements where a pure transition and the knowledge of its orientation relative to the molecular framework is key. ${ }^{[1 \mathrm{a}]}$

All absorption spectra display a small shoulder at longer wavelengths which can be attributed to a residual protonation of the structure that persists under neutral conditions as shown by the $\mathrm{pH}$ titration experiments (vide infra and Figure S5). This shoulder is more pronounced in the case of qAN3, suggesting that the protonated species is present to a larger extent, possibly partly explaining why the molar absorptivity of qAN3 in the principal lower absorption band is slightly weaker than that of its sister compounds and what was expected based on the calculations. In solvents other than water this shoulder is not observed (Figure S2), further indicating a protonated or tautomeric structure that only forms in water. The absorption spectra of the qAN derivatives recorded in toluene, DCM, DMSO, methanol and ethanol show only minor differences when compared with the spectra in water (Figure S2). Slight variations in the molar absorptivities are observed but the maximum absorption wavelengths remain essentially the same, showing that the ground state is not highly sensitive to the polarity of the solvent. As predicted by the TDDFT calculations, the qAN derivatives display improved absorptive properties compared to the parent compound qA.

Table 3. Optical properties of the qAN derivatives in milliQ water at room temperature

\begin{tabular}{llllllllll}
\hline & $\lambda_{\text {abs }}$ & $\varepsilon$ & $\lambda_{\mathrm{em}}$ & $\Phi_{\mathrm{F}}$ & $\mathrm{f}_{\mathrm{S} 0 \rightarrow \mathrm{S} 1}$ & $\varepsilon \cdot \Phi_{\mathrm{F}}$ & $\left\langle\tau_{\mathrm{F}}\right\rangle$ & $\mathrm{k}_{\mathrm{r}}$ & $\mathrm{k}_{\mathrm{nr}}$ \\
\hline qA & 335 & 5000 & 456 & 0.07 & 0.060 & 340 & 3.2 & 2.13 & 2.91 \\
qAN1 & 360 & 9500 & 430 & 0.18 & 0.20 & 1700 & 4.8 & 3.76 & 1.71 \\
qAN2 & 340 & 9450 & 530 & 0.06 & 0.041 & 576 & 3.8 & 1.61 & 2.47 \\
qAN3 & 330 & 5900 & 510 & 0.01 & 0.035 & 59 & 1.4 & 0.71 & 7.07 \\
qAN4 & 356 & 7300 & 445 & 0.32 & 0.30 & 2300 & 4.8 & 6.67 & 1.42 \\
\hline
\end{tabular}

Maximum absorption wavelength $\left(\lambda_{\text {abs }}\right)$ in $\mathrm{nm}$, molar absorptivity $(\varepsilon)$ in $\mathrm{M}^{-1} \cdot \mathrm{cm}^{-1}$, maximum emission wavelength $\left(\lambda_{\mathrm{em}}\right)$ in $\mathrm{nm}$, fluorescence quantum yield $\left(\Phi_{\mathrm{F}}\right)$ and average fluorescence lifetime $\left(<\tau_{F}>\right)$ in ns. Radiative and non-radiative decay rates $\left(\mathrm{k}_{\mathrm{r}}\right.$ in $10^{7} \mathrm{~s}^{-1}$ and $\mathrm{k}_{\mathrm{nr}}$ in $10^{8} \mathrm{~S}^{-1}$ ) calculated using the measured fluorescence quantum yield and lifetime values. $\mathrm{f}_{\mathrm{s} 0 \rightarrow \mathrm{s} 1}$ included as comparison.

\section{Steady-state fluorescence properties}

The fluorescence emission of qAN1 and qAN4 were measured in different solvents and found to be slightly sensitive to the polarity of the solvent used, with a gradual red-shift of $20 \mathrm{~nm}$ for qAN1 and $35 \mathrm{~nm}$ for qAN4 when switching from toluene to water (Figure 3). In water, both qAN1 and qAN4 exhibit strong fluorescence compared to $\mathrm{qA}\left(\Phi_{\mathrm{F}}=6.8 \%\right)$ with maxima at 430 and $445 \mathrm{~nm}$ (Figure 3) and high quantum yields of $18 \%$ and $32 \%$, respectively (Table 3 ). The increase in measured quantum yields is consistent with the increased predicted oscillator strengths according to the Strickler-Berg equation (Table 3). ${ }^{[21]}$

Despite their red-shifted absorption, the emission maxima of qAN1 and qAN4 are blue-shifted compared to that of qA due to smaller Stokes shifts (121 nm for qA vs. 70 and $89 \mathrm{~nm}$ for qAN1 and qAN4, respectively; Table 3). However, the absorption and emission spectra of qAN1 and qAN4 remain well separated thus minimizing possible effects of self-quenching. The emission energies in the different solvents display a fairly linear relation with the solvent polarity according to the $\mathrm{E}_{\mathrm{T}}(30)$ solvent polarity scale $^{[24]}$ (Figure S3) with the exception of DMSO.
The quantum yields range from $13 \%$ in methanol to $38 \%$ in DMSO for qAN1 and from $12 \%$ in DCM to $49 \%$ in DMSO for qAN4 (Table S1). Despite the fact that they are both most fluorescent in DMSO, the solvent-dependence of the quantum yield varies between the two compounds and displays no particular trend with regard to polarity. To better understand and investigate the effect of tautomerization a methylated version of qAN1 (MeqAN1) was synthesized, in which the secondary amine function in the middle ring of qAN1 is methylated (see supplementary information). In this analogue, the possibility for prototropic tautomerism is eliminated and, since the absorptive and emissive properties of qAN1 and its methylated counterpart are very similar (Figure S8), it implies that the preferred tautomer of qAN1 is indeed the one depicted in Figure 1. This information is highly relevant since this tautomer is the form that would base-pair with thymine in duplex nucleic acid structures.

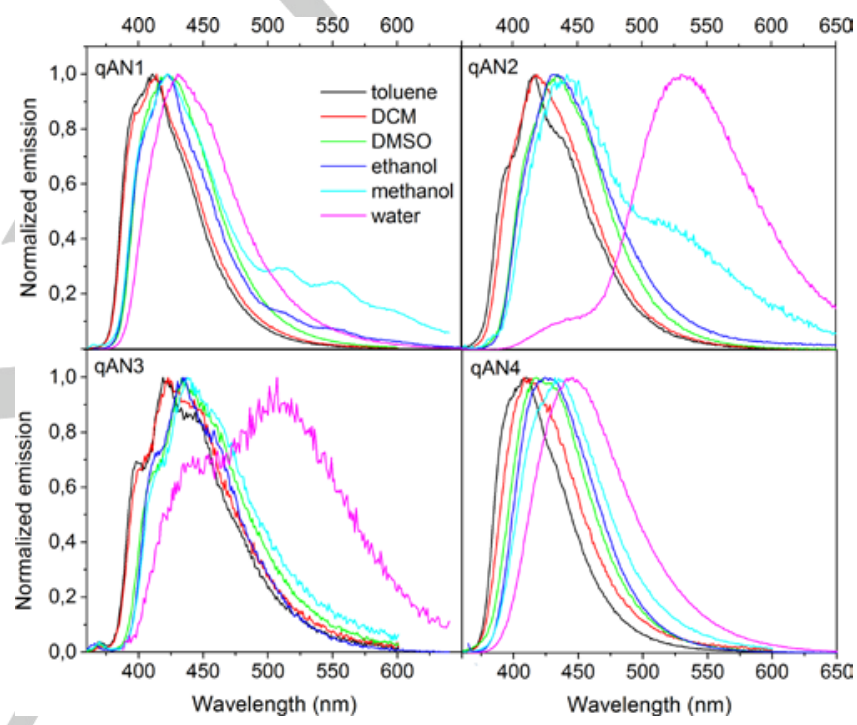

Figure 3. Normalized fluorescence emission spectra of the qAN family in various solvents.

As expected from the weaker predicted $S_{0} \rightarrow S_{1}$ oscillator strengths and the Strickler-Berg relation, ${ }^{[21]}$ qAN2 and qAN3 have lower fluorescence quantum yields of $6.1 \%$ and $1.0 \%$, respectively. They display the same linear increase of the maximum emission wavelengths with the polarity from toluene to methanol. In water however, both qAN2 and qAN3 display dualband emissions, with the two maxima around 450 and $550 \mathrm{~nm}$, the latter being predominant (Figure 3). The $450 \mathrm{~nm}$ peak corresponds well with those observed for qA, qAN1 and qAN4 and obeys the linear relationship between emission energy and polarity (Figure S3). However, this relationship is not satisfied if we take into account the main red-shifted peak suggesting that it does not originate from a simple polarity effect but more likely results from the presence of a different species.

The fluorescence and absorption of qAN2 were also measured at different $\mathrm{pH}$ values (Figure S5). At pH 2 qAN2 displays a single emission band at $525 \mathrm{~nm}$ but when increasing the $\mathrm{pH}$ to 7 , a band appears at $425 \mathrm{~nm}$ while the $525 \mathrm{~nm}$ band 
decreases in intensity but still remains the major band. This suggests that the emission of qAN2 - and most likely of qAN3 in water originates from a combination of a minor unprotonated species and a major protonated one that is formed in the excited state even at neutral $\mathrm{pH}$ when the ground state is not protonated the absorption spectrum being indeed the one of the unprotonated form.

Excited-state intra- or inter-molecular proton transfer is a common phenomenon due to the modification of the pKa of a molecule in its excited-state. ${ }^{[25]}$ In fluorophores, proton transfer usually results in large modification of the emissive properties depending on the polarity and protic character of the solvent. ${ }^{[26]}$ The excited-state proton transfer for qAN2 is most likely an intermolecular process since it is not observed in aprotic solvent. However, the proton transfer is also highly dependent on polarity since it is does not occur in ethanol and only to a small extent in methanol as attested to by the relative intensities of the two emission bands. To further investigate the polarity dependence of the proton transfer, the fluorescence of qAN2 in water-ethanol mixtures was measured (Figure S6). When the percentage of water was below $50 \%$ by volume almost no proton transfer was observed but above this threshold the second red-shifted emission band due to the proton transfer is visible and its intensity increases with the water content i.e. the polarity.

Due to this excited-state proton transfer qAN2 could be used as a dual-emission polarity probe, even if this is only observed over a narrow polarity range in water and ethanol mixtures. The polarity of the environment of the nucleobases depends on DNA hybridization and, thus, a probe with a polarity-sensitive dualemission could be of particular interest to monitor changes in the DNA structure occurring e.g. upon protein- or drug-interaction. FBAs based on the 3-hydroxychromone scaffold with similar polarity-dependent dual emission properties originating from an excited-state intramolecular proton transfer were developed by Burger and coworkers and successfully applied to probe DNAprotein interactions. ${ }^{[27]}$ These base analogues are not however specific and they slightly destabilize the DNA duplex structure.

The replacement of the benzene ring with a pyridine in the quadracyclic scaffold yields very different results in terms of fluorescence brightness $\left(\varepsilon \cdot \Phi_{\mathrm{F}}\right)$, depending on the position of the nitrogen. With similar fluorescence quantum yields, qAN3 presents no significant improvement compared to qA but qAN2 is brighter due to its increased absorptivity $\left(\varepsilon \cdot \Phi_{\mathrm{F}}=576\right)$. For qAN1 and qAN4, the combined improvement of the absorptivity and fluorescence quantum yields results in highly increased brightness values ( $\varepsilon \cdot \Phi_{\mathrm{F}}=1700$ and 2300 respectively).

\section{Time-resolved fluorescence properties}

In anticipation of future use of the qAN derivatives inside nucleic acids in anisotropy and FRET-measurements we decided to study time-resolved fluorescence (Figure S4, Table S2). The fluorescence decays of qAN1 and qAN4 in water were measured at two different emission wavelengths and were accurately fitted to a monoexponential function. The resulting single lifetime of 4.8 ns for both qAN1 and qAN4 (Table 3) is insensitive to the emission wavelength and is in a very good regime for future time-resolved measurements in DNA. The average fluorescence lifetimes of qAN2 and qAN3 (Table 3) were obtained by fitting their fluorescence decays to biexponential functions (Table S2). The average lifetime of qAN3 (1.4 ns) is much lower than those of qAN1 and qAN4, which is consistent with its lower quantum yield, while that of qAN2 (3.76 ns) is closer to the corresponding values for qAN1 and qAN4.

The radiative $\left(\mathrm{k}_{\mathrm{r}}\right)$ and non-radiative $\left(\mathrm{k}_{\mathrm{nr}}\right)$ decay rates were calculated using the relations $\Phi_{\mathrm{F}}=\mathrm{k}_{\mathrm{r}} /\left(\mathrm{k}_{\mathrm{r}}+\mathrm{k}_{\mathrm{nr}}\right)$ and $\tau_{\mathrm{F}}=1 /\left(\mathrm{k}_{\mathrm{r}}+\right.$ $\mathrm{k}_{\mathrm{nr}}$ ) (Table 3). As expected considering their similar rigid aromatic structures, the four qAN derivatives and qA have comparable non-radiative decay rates possibly due to close similarities in non-radiative deactivation pathways. As a direct consequence, due to the relation between $f\left(S_{0} \rightarrow S_{1}\right)$ and $k_{r}$ through the Strickler-Berg relation, ${ }^{[21]}$ the oscillator strengths of their $\mathrm{S}_{0} \rightarrow \mathrm{S}_{1}$ transitions account very well for the relative values of the quantum yields (Table 3 ). There is a minor exception with qAN3 which shows a slightly higher non-radiative decay rate and thus a fluorescence quantum yield lower than the quantum chemical calculations of the oscillator strength would suggest.

\section{Absorption and fluorescence properties of qAN1 and qAN4 in acidic $\mathrm{pH}$}

The qAN derivatives possess four heterocyclic nitrogen atoms and one secondary amine capable of participating in acidbase equilibria. We therefore decided to study the influence of $\mathrm{pH}$ on the absorption as well as on the steady-state and timeresolved fluorescence properties of the most promising fluorescent base analogue candidates qAN1 and qAN4. The two compounds behave quite similarly and, as expected, several different equilibria are observed. Focusing on acidic conditions, we were able to isolate one acid-base equilibrium with two isosbestic points in the UV region of the absorption spectrum (Figure 4).
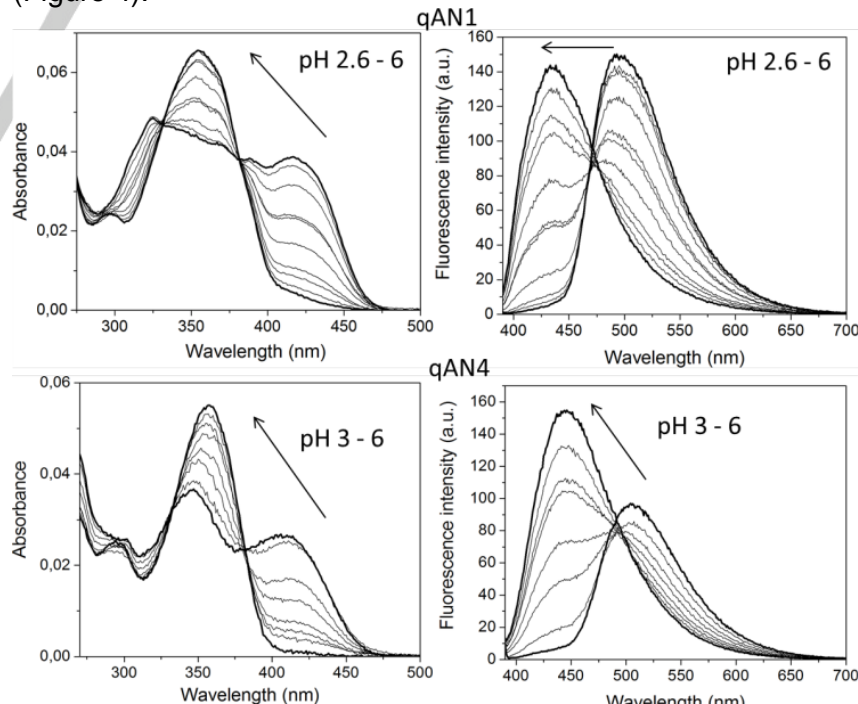

Figure 4. Absorption and emission of qAN1 and qAN4 at $7 \mu \mathrm{M}$ measured at room temperature in different buffers with $\mathrm{pH}$ varying from 2.6 to 6 (arrows indicate increase in $\mathrm{pH}$ ). 
Interestingly enough, we observe a dual emission depending on $\mathrm{pH}$ much more pronounced than in the case of qAN2 (vide supra and Figure S5), with a clearly distinct red-shifted emission band appearing at acidic $\mathrm{pH}\left(\lambda_{\mathrm{em}} \approx 505 \mathrm{~nm}\right)$. By plotting the intensity ratio of the protonated form over the neutral form versus $\mathrm{pH}$, we were able to calculate the $\mathrm{pK}_{\mathrm{a}}$ of $\mathrm{qAN} 1$ $\left(\mathrm{pK}_{\mathrm{a}}=4.3\right)$ with similar results using either absorbance or fluorescence intensity (Figure S7). The $\mathrm{pK}_{\mathrm{a}}$ of qAN4 could not accurately be calculated by the same method since the different equilibria overlap, and the corresponding titration curve could not be correctly fitted, at least not with a simple sigmoid curve, but was estimated to be around 4.5. The fluorescence decays of qAN1 and qAN4 in a pH 3 buffer could both be fitted to a single exponential decay function (Table S2, Figure S4) strongly suggesting that we virtually only have one species present at $\mathrm{pH}$ 3. Compared to the values in milliQ water the lifetime of qAN1 increased from $4.8 \mathrm{~ns}$ to $5.4 \mathrm{~ns}$ while that of qAN4 decreased from 4.8 ns to 3.92 ns.

Just as qAN2 has the potential to be used as a polarity probe, the dual emissions of qAN1 and qAN4 could be used for ratiometric fluorescent sensing of $\mathrm{pH}$. Moreover, the acidic and neutral forms are associated with two distinct fluorescence lifetimes that potentially can be used in fluorescence lifetime imaging microscopy (FLIM) for sensing intracellular $\mathrm{pH}$ for instance. ${ }^{[28]}$ In particular the $\mathrm{pK}_{\mathrm{a}}$ of qAN1 is perfectly suited to monitor $\mathrm{pH}$ changes in acidic cellular vesicles e.g. lysosomes or endosomes. ${ }^{[29]}$ However, before any utilization of the qAN derivatives inside cells can be imagined, their water solubility has to be significantly improved. For instance this can be achieved by replacing the ethyl group (Figure 1) by short PEG chains or other hydrophilic substituents. Another plausible modification to increase the solubility would be the nucleosidic form which we already plan to synthesize for our future investigations of the qAN derivatives inside nucleic acid systems.

\section{Conclusions}

Building on the existing quadracyclic adenine scaffold of qA, which has been shown to be an unperturbing and specific adenine analogue inside DNA with fluorescent properties comparable to 2-AP, quantum chemical calculations were successfully used to design four new potential adenine analogues, qAN1, qAN2, qAN3 and qAN4 with improved absorptive and emissive properties. The four compounds were synthesized via an efficient pathway from a common intermediate utilizing the Suzuki-Miyura coupling reaction as the key step in the sequence.

The photophysical properties of the qAN derivatives, position and strengths of the absorption bands, as well as fluorescence quantum yields via the oscillator strengths $\left(f_{\mathrm{s}_{0} \rightarrow \mathrm{s}_{1}}\right)$ and the Strickler-Berg relation, were very well predicted by the TDDFT calculations. Two of the compounds qAN1 and qAN4 display significantly increased molar absorptivities and fluorescence quantum yields resulting in high brightnesses $\left(\varepsilon \cdot \Phi_{\mathrm{F}}=1700\right.$ and 2300 , respectively) with up to a 7-fold increase for qAN4 compared to qA. The other two derivatives, qAN2 and qAN3 display lower quantum yields, due to a weak $S_{0} \rightarrow S_{1}$ transition and undergo an excited-state proton transfer reaction resulting in dual-emission spectra in water with a main peak red-shifted $100 \mathrm{~nm}$ compared to the other quadracyclic adenines.

The high correlation between the measured quantum yields and the predicted oscillator strength in the qA series shows that rational design of high quantum yield fluorophores is possible. However, such a design approach is restricted to series of compounds where structural similarities are high and where virtually constant non-radiative decay rates can be anticipated. In such cases it is possible to design the best candidates in silico before committing huge amounts of time and resources to the actual synthesis of novel compounds.

The brightest compounds, qAN1 and qAN4, will most likely display better optical properties than qA inside DNA and are thus very promising fluorescent adenine analogues. Despite their lower fluorescence quantum yields, qAN2 and qAN3 still display interesting properties, in particular qAN2 whose brightness $\left(\varepsilon \cdot \Phi_{\mathrm{F}}=576\right)$ while being lower than that of qAN1 and qAN4 is still higher than that of qA. More importantly, its ability to form a protonated excited state resulting in a polarity sensitive dual-band emission, could prove very useful to investigate DNA structural changes induced by DNA-protein and DNA-drug interactions. The $\mathrm{pH}$ dependent dual-emission of qAN1 and qAN4 could be exploited in a similar fashion and expands the potential field of application of the qAN compounds. Solvatochromism is a common trait of fluorescent base analog or base surrogates and is used to monitor changes in the local probe environment due to hybridization state or abasic sites for instance. ${ }^{[30]} \mathrm{A}$ few examples of $\mathrm{pH}$ sensitive base analogs have also been reported. ${ }^{[30 b, 31]}$ In most of these examples however, changes in the environment of the probe results in shifts of maximum emission wavelength and/or changes in fluorescence intensity. Wavelength-ratiometric probes, like the ones presented here, are more seldom and, since the absolute fluorescence intensity is dependent on the instrumentation and on various quenching effects that are often unaccounted for, they show increased sensitivity and are preferable to purely intensity-based probes. ${ }^{[32]}$ During the various biological processes it is subjected to, DNA is exposed to different environments, with varying $\mathrm{pH}$ and polarity, and it is plausible that once incorporated into DNA, the qAN derivatives will be able to probe these events. The synthesis of the nucleosides of this series of compounds is currently ongoing and we eagerly look forward to investigating their behavior inside nucleic acid systems.

\section{Experimental Section}

\section{General methods}

Reagents were purchased from various chemical vendors with a minimum purity of $95 \%$ and were used as delivered without further purification. All solvents used for reactions were purchased dry in SureSeal bottles from Sigma-Aldrich. All other used solvents were purchased as CHROMASOLV grade from Sigma-Aldrich. For the chemical synthesis distilled water was 
utilized throughout for all procedures. Microwave reactions were performed using a Biotage Initiator Microwave synthesizer, using single mode microwave irradiation with temperature and pressure control and with fixed hold time on. Reactions were monitored by TLC performed on Merck silica gel $60 \mathrm{~F}_{254}$ coated glass plates and visualized under UV light at $254 \mathrm{~nm}$. UPLC-MS (ESI/UV) analysis was performed using a Waters Aquity UPLCMS System equipped with either a Waters ACQUITY UPLC ${ }^{\circledR}$ HSS C18 column $1.8 \mu \mathrm{m}$, length $50 \mathrm{~mm}$, ID $2.1 \mathrm{~mm}$ running a gradient of $\mathrm{H}_{2} \mathrm{O}-\mathrm{MeCN}(95: 5)$ to $\mathrm{H}_{2} \mathrm{O}-\mathrm{MeCN}$ (5:95), with the $\mathrm{H}_{2} \mathrm{O}$ eluent containing $1 \%$ formic acid $(\mathrm{pH} 3)$ or a Waters ACQUITY UPLC $®$ BEH C18 column $1.7 \mu \mathrm{m}$, length $50 \mathrm{~mm}$, ID $2.1 \mathrm{~mm}$ running a gradient of $\mathrm{H}_{2} \mathrm{O}-\mathrm{MeCN}(95: 5)$ to $\mathrm{H}_{2} \mathrm{O}-\mathrm{MeCN}(5: 95)$, with the $\mathrm{H}_{2} \mathrm{O}$ eluent containing $1 \%$ Ammonium hydroxide $(\mathrm{pH}$ 10). The chromatograms were analysed with MassLynx Software. Flash chromatography was performed on a Biotage SP-1 instrument using SNAP (KP-Sil or KP-NH) cartridges. HPLC purification was performed with formic acid as modifier on a Gilson Preperative HPLC system with a GX-281 liquid handler, UV/Vis-155 detector, 333/334 binary master pump system and an Xbridge C18 $10 \mu \mathrm{m} 250 \times 50 \mathrm{~mm}$ column controlled by a computer running Trilution LC software. Freeze drying was performed on a Christ Alpha 2-4 Freeze Dryer. ${ }^{1} \mathrm{H}$ NMR were recorded on either a Bruker AV500 (500 MHz) system equipped with a CryoProbe or a Bruker AV600 (600 MHz) system. All shifts are reported as ppm relative to the deuterated solvent $\left(\mathrm{CDCl}_{3}, \quad[\mathrm{D}]_{6}-\mathrm{DMSO}\right)$. MestRenova 8.1 was used for interpretation of all NMR spectra.

\section{Suzuki-Miyaura Cross-Coupling Reaction Condition Screen}

A range of litterature procedures were tested out ${ }^{[46-55]}$ in order to find suitable conditions for the Suzuki-Miyaura cross-coupling reaction between starting material 3 and anilines $\mathbf{4 a}$ and $\mathbf{4 e}$. All reactions were run at $80{ }^{\circ} \mathrm{C}$ for 24 hours and conversion determined by by UPLC-MS analysis.

\section{General method A: Suzuki-Miyaura Cross-Coupling}

A reaction vial with a magnetic stir bar was charged with compound 3 (200 mg, $0.65 \mathrm{mmol}$ ), iodopyridine substrate $\mathbf{4 a - 4 d}$ (158 $\mathrm{mg}, 0.72 \mathrm{mmol}$ ), potassium phosphate (346 mg, 1.63 $\mathrm{mmol}$ ) and bis(triphenylphosphine)palladium(II) dichloride (23 $\mathrm{mg}, 0.019 \mathrm{mmol})$. The vial was sealed with a septum, evacuated and flushed with $\mathrm{N}_{2}$ three times. Then $\mathrm{MeCN}(2 \mathrm{~mL})$ and water $(2 \mathrm{~mL})$ was added, and the vial was heated to $80^{\circ} \mathrm{C}$ for 2 hours. After cooling to $\mathrm{RT}$, the reaction mixture was partitioned between EtOAc $(25 \mathrm{~mL})$ and water $(25 \mathrm{~mL})$, and the water phase was extracted with EtOAc $(3 \times 25 \mathrm{~mL})$. The combined organic phases were washed with brine $(30 \mathrm{~mL})$, dried over $\mathrm{MgSO}_{4}$ and the volatiles were removed in vacuo. The residue was absorbed onto Celite $\AA$ and purified by flash chromatography to yield the desired product.

\section{General procedure B: Cyclisation by $\mathrm{S}_{\mathrm{N}} \mathrm{Ar}$}

An oven-dried reaction vial equipped with a magnetic stir bar was charged with compound 5a-5d (1 eq) dissolved in THF $(0.05 \mathrm{M})$ under $\mathrm{N}_{2}$ atmosphere and was sealed with a septum.
Chlorotrimethylsilane (1.05 eq) was added dropwise using a gas-tight syringe and the reaction was stirred at RT for $30 \mathrm{~min}$ before lithium bis(trimethylsilyl)amide ( $2.5 \mathrm{eq}$ ) was then added dropwise. The reaction was heated to $100^{\circ} \mathrm{C}$ for $3 \mathrm{~h}$ on a heating block. The reaction was quenched with water $(5 \mathrm{~mL})$ and extracted with DCM $(3 \times 15 \mathrm{~mL})$. The combined organic phases were washed with brine $(10 \mathrm{~mL})$, dried over $\mathrm{MgSO}_{4}$, and the volatiles were removed in vacuo. The crude was purified either by flash chromatography (DCM:MeOH 100:0 - 98:2) or by HPLC.

\section{Photophysical measurements:}

The quadracylic adenine compounds were dissolved in DMSO to form stock solutions of concentrations between 1 and $10 \mathrm{mM}$. Measurements were performed in $1 \mathrm{~cm}$ pathlength quartz cuvettes at concentrations in the $\mu \mathrm{M}$ range. The DMSO content of the working solutions was usually $0.1 \%$ and never exceeded 1\%. Absorption spectra were recorded on a Varian Cary 5000 spectrophotometer. Molar absorption coefficients were determined by Lambert-Beer's law. All measurements were duplicated. Fluorescence spectra were recorded on a Horiba Spex fluorolog 3 (excitation and emission slits were $2 \mathrm{~nm}$ ) or on a Cary Eclipse fluorimeter (excitation and emission slits were 5 $\mathrm{nm})$. Spectra were corrected for variations of the detector sensitivity and Raman scattering.

Fluorescence quantum yields were determined relative to quinine sulfate $\left(\Phi_{\mathrm{F}}=0.55\right)$ in $0.5 \mathrm{M} \mathrm{H}_{2} \mathrm{SO}_{4}$ at room temperature $\left(20^{\circ} \mathrm{C}\right) .{ }^{[33]}$ The same excitation wavelength of $350 \mathrm{~nm}$ was used for all the samples and the reference. In aqueous solution (water and acidic buffers), quantum yields were calculated by measuring the absorption and fluorescence of 5 solutions of different concentrations $(O D<0.05)$. After verifying the linearity between absorbance and integrated fluorescence intensity, the fluorescence quantum yield $\Phi$ was given by the following relation:

$$
\Phi_{\mathrm{s}}=\Phi_{\mathrm{ref}} \frac{\mathrm{A}_{r e f} F_{s}}{A_{s} F_{r e f}}\left(\frac{\eta_{s}}{\eta_{r}}\right)^{2}
$$

where $A$ is the absorption at the excitation wavelength $(350 \mathrm{~nm})$, $F$ the integrated fluorescence intensity, $\eta$ is the refractive index of the solvent and $s$ refers to the sample and ref to the reference. Measurements were duplicated. In other solvents, quantum yields were roughly estimated by a one point measurement. Buffers for $\mathrm{pH}$ titrations: glycine- $\mathrm{HCl}(\mathrm{pH} 2-2.5)$, citrate buffer ( $\mathrm{pH} 2.6-6.5)$, phosphate buffer ( $\mathrm{pH} 7-8)$.

Time-resolved fluorescence decays were measured using TCSPC (time-correlated single photon counting). The excitation source was a $377 \mathrm{~nm}$ laser diode pulsed at $10 \mathrm{MHz}$. The fluorescence emission was filtered by a monochromator with a resolution of $10 \mathrm{~nm}$ and detected by a microchannel plate photomultiplier Hamamatsu R3809U-50. The counts were fed into a multichannel analyzer with 2048 channels (Life-spec, Edinburgh Analytical Instruments) where a maximum of 10000 counts were recorded in the top channel. All fluorescence decays were recorded in a time window of 100 ns. The data 
were convoluted with the instrument response function and fitted to mono- or bi-exponential functions using Fluofit Pro v.4 (PicoQuant $\mathrm{GmbH}$ ). The average lifetimes were amplitudeweighted.

\section{Quantum chemical calculations}

All DFT geometry optimisations were performed in the groundstate of the molecule using the B3LYP functional[ ${ }^{[34]}$ as implemented in Gaussian09. ${ }^{[35]}$ Electronic excitations were calculated using TDDFT ${ }^{[36]}$ B3LYP/6-311+G(2d) as implemented in Gaussian09. Solvation effects were mimicked by applying a CPCM solvation shel|[37] in the TDDFT calculations. Restricted Hartree-Fock (RHF) wavefunctions were used in all calculations.

\section{Acknowledgements}

The authors thank Carl Tryggers Stiftelse (M.G.), Stiftelsen Olle Engkvist Byggmästare (L.M.W.) and the Swedish Research Council 2013-4375 (L.M.W.) for financial support.

Keywords: fluorescent probes $\bullet$ modified nucleobases • aromatic substitutions $\bullet \mathrm{C}-\mathrm{C}$ coupling $\bullet$ TDDFT calculations

[1] a) S. Preus, L. M. Wilhelmsson, ChemBiochem 2012, 13, 1990-2001; b) B. Juskowiak, Anal. Bioanal. Chem. 2011, 399, 3157-76; c) A. S. Boutorine, D. S. Novopashina, O. A. Krasheninina, K. Nozeret, A. G. Venyaminova, Molecules., 2013,.18, 15357-15397.

[2] A. P. Demchenko, Introduction to Fluorescence Sensing, Springer, 2009.

[3] J. Widengren, V. Kudryavtsev, M. Antonik, S. Berger, M. Gerken, C. A. M. Seidel, Anal. Chem. 2006, 78, 2039-2050.

[4] M. Daniels, W. Hauswirth, Science 1971, 171, 675-677.

[5] a) R. W. Sabnis, Handbook of Biological Dyes and Stains: Synthesis and Industrial Applications, Wiley, 2010; b) M. Vendrell, D. Zhai, J. C. Er, Y.-T. Chang, Chem. Rev. 2012, 112, 4391-4420.

[6] S. Kalinin, T. Peulen, S. Sindbert, P. J. Rothwell, S. Berger, T. Restle, R. S. Goody, H. Gohlke, C. A. M. Seidel, Nat. Methods 2012, 9, 1218-1225.

[7] a) K. Gislason, S. T. Sigurdsson, Bioorg. Med. Chem. Lett. 2013, 23, 264-267; b) B. J. Rodgers, N. A. Elsharif, N. Vashisht, M. M. Mingus, M. A. Mulvahill, G. Stengel, R. D. Kuchta, B. W. Purse, Chem. Eur. J. 2013, 20, 2010-2015; c) M. Segal, B. Fischer, Org. Biomol. Chem. 2012, 10, 1571-1580; d) M. Suchý, R. H. E. Hudson, J. Org. Chem. 2014, 79, 3336-3347; e) A. Okamoto, Y. Saito, I. Saito, J. Photochem. Photobiol. C 2005, 6, 108-122; f) K. Seio, T. Kanamori, M. Tokugawa, H. Ohzeki, Y. Masaki, H. Tsunoda, A. Ohkubo, M. Sekine, Bioorg. Med. Chem. 2013, 21, 3197-3201 g) A. Dumas, N. W. Luedtke, ChemBiochem 2011, 12, 2044-2051; h) X. Ming, F. Seela, Chem. Eur. J. 2012, 18, 9590-9600; i) M. Weinberger, F. Berndt, R. Mahrwald, N. P. Ernsting, H.-A Wagenknecht, J. Org. Chem. 2013, 78, 2589-2599.

[8] M. Winnacker, E. T. Kool, Angew. Chem. Int. Ed. Engl. 2013, 52, 1249812508.

[9] Y. N. Teo, E. T. Kool, Chem. Rev. 2012, 112, 4221-4245.

[10] a) J. N. Wilson, E. T. Kool, Org. Biomol. Chem. 2006, 4, 4265-4274; b) D. W. Dodd, R. H. E. Hudson, Mini. Rev. Org. Chem. 2009, 6, 378-391; c) R. W. Sinkeldam, N. J. Greco, Y. Tor, Chem. Rev. 2010, 110, 2579-2619; d) L. M. Wilhelmsson, Q. Rev. Biophys. 2010, 43, 159-183.

[11] a) K. Lang, R. Rieder, R. Micura, Nucleic Acids Res. 2007, 35, 53705378; b) T. Ono, S. K. Edwards, S. Wang, W. Jiang, E. T. Kool, Nucleic Acids Res. 2013, 41, e127; c) V. Posse, E. Hoberg, A. Dierckx, S. Shahzad, C. Koolmeister, N.-G. Larsson, L. M. Wilhelmsson, B. M. Hällberg, C. M. Gustafsson, Nucleic Acids Res. 2014, 1-10; d) S.
Srivatsan, N. Greco, Y. Tor, Angew. Chemie 2008, 47, 6661-6665; e) K. M. Rankin, M. Sproviero, K. Rankin, P. Sharma, S. D. Wetmore, R. a Manderville, J. Org. Chem. 2012, 77, 10498-508.

[12] L. H. Yuen, R. M. Franzini, S. Wang, P. Crisalli, V. Singh, W. Jiang, E. T. Kool, Angew. Chem. Int. Ed. Engl. 2014, 53, 5361-5365.

[13] P. Sandin, J. Tumpane, K. Börjesson, L. M. Wilhelmsson, T. Brown, B. Nordén, B. Albinsson, P. Lincoln, J. Phys. Chem. C 2009, 113, 59415946.

[14] D. C. Ward, E. Reich, L. Stryer, J. Biol. Chem. 1969, 244, 1228-1237.

[15] a) J. R. Barrio, J. Secrist, N. J. Leonard, Proc. Natl. Acad. Sci. U. S. A 1972, 69, 2039-2042; b) J. A. Secrist, J. R. Barrio, N. J. Leonard, Science 1972, 175, 646-647.

[16] a) K. Börjesson, S. Preus, A. H. El-Sagheer, T. Brown, B. Albinsson, L. M. Wilhelmsson, J. Am. Chem. Soc. 2009, 131, 4288-4293; b) S. Preus, K. Kilså, F.-A. Miannay, B. Albinsson, L. M. Wilhelmsson, Nucleic Acids Res. 2013, 41, e18; c) P. Sandin, K. Börjesson, H. Li, J. Mårtensson, T. Brown, L. M. Wilhelmsson, B. Albinsson, Nucleic Acids Res. 2008, 36, 157-167; d) S. Preus, K. Börjesson, K. Kilså, B. Albinsson, L. M. Wilhelmsson, J. Phys. Chem. B 2010, 114, 1050-1056.

[17] a) A. Dierckx, P. Dinér, A. H. El-Sagheer, J. D. Kumar, T. Brown, M. Grøtli, L. M. Wilhelmsson, Nucleic Acids Res. 2011, 39, 4513-4524; b) C. P. Lawson, A. Dierckx, F.-A. Miannay, E. Wellner, L. M. Wilhelmsson, M. Grøtli, Org. Biomol. Chem. 2014, 12, 5158-5167; c) C. Dyrager, K. Börjesson, P. Dinér, A. Elf, B. Albinsson, L. M. Wilhelmsson, M. Grøtli, Eur. J. Org. Chem. 2009, 2009, 1515-1521.

[18]A. Dierckx, F.-A. Miannay, N. Ben Gaied, S. Preus, M. Björck, T. Brown, L. M. Wilhelmsson, Chem. Eur. J. 2012, 18, 5987-5897.

[19] A. Dallmann, L. Dehmel, T. Peters, C. Mügge, C. Griesinger, J. Tuma, N. P. Ernsting, Angew. Chem. Int. Ed. Engl. 2010, 49, 5989-5992.

[20] S. Preus, K. Kilså, L. M. Wilhelmsson, B. Albinsson, Phys. Chem. Chem. Phys. 2010, 12, 8881-8892.

[21] S. J. Strickler, R. A. Berg, J. Chem. Phys. 1962, 37, 814-822

[22] a) J. Streling, in MSc Thesis Gothenburg University, 2012; b) N. Kudo, M. Perseghini, G. C. Fu, Angew. Chem. Int. Ed. 2006, 45, 1282-1284; c) J. Yang, S. Liu, J.-F. Zheng, J. Zhou, Eur. J. Org. Chem. 2012, 2012, 62486259; d) L. Zhang, Z. Li, X.-j. Wang, N. Yee, C. H. Senanayake, Synlett 2012, 23, 1052-1056; e) C. P. Ashcroft, S. J. Fussell, K. Wilford Tetrahedron Lett. 2013, 54, 4529-4532; f) F. Proutiere, M. Aufiero, F. Schoenebeck, J. Am. Chem. Soc. 2011, 134, 606-612; g) C. Bengtsson, F. Almqvist, J. Org. Chem. 2009, 75, 972-975; h) N. Kataoka, Q. Shelby, J. P. Stambuli, J. F. Hartwig, J. Org. Chem. 2002, 67, 5553-5566; i) K. L. Billingsley, K. W. Anderson, S. L. Buchwald, Angew. Chem. Int. Ed. 2006 45, 3484-3488; j) J. Z. Deng, D. V. Paone, A. T. Ginnetti, H. Kurihara, S. D. Dreher, S. A. Weissman, S. R. Stauffer, C. S. Burgey, Org. Let. 2008, 11, 345-347.

[23] S. Rajeswari, R.J. Jones, M.P. Cava, Tetrahedron Lett., 1987, 28,. 50995102.

[24] C. Reichardt, Chem. Rev. 1994, 94, 2319-2358.

[25] a)H. Shizuka, Acc. Chem. Res. 1985, 141-147; b) L. M. Tolbert, K. M. Solntsev, Acc. Chem. Res. 2002, 35, 19-27.

[26] a) I. Presiado, Y. Erez, R. Gepshtein, D. Huppert, J. Phys. Chem. C 2010, 114, 3634-3640; b) Y. Erez, D. Huppert, J. Phys. Chem. A 2010, 114, 8075-8082; c) M. Quick, A. Weigel, N. P. Ernsting, J. Phys. Chem. B 2013, 117, 5441-5447; d) M. Zahid, G. Grampp, A. Mansha, I. A. Bhatti, S. Asim, J. Fluoresc. 2013, 23, 829-37.

[27] a) D. Dziuba, V. Y. Postupalenko, M. Spadafora, A. S. Klymchenko, V. Guérineau, Y. Mély, R. Benhida, A. Burger, J. Am. Chem. Soc. 2012, 134, 10209-10213; b) D. Dziuba, I. a Karpenko, N. P. F. Barthes, B. Y. Michel, A. S. Klymchenko, R. Benhida, A. P. Demchenko, Y. Mély, A. Burger, Chem. Eur. J. 2014, 20, 1998-2009.

[28] a) M. Y. Berezin, S. Achilefu, Chem. Rev. 2010, 110, 2641-2684; b) H. J. Park, C. S. Lim, E. S. Kim, J. H. Han, T. H. Lee, H. J. Chun, B. R. Cho, Angew. Chem. Int. Ed. Engl. 2012, 51, 1-5.

[29]H. M. H. J. Kim, C. H. Heo, H. M. H. J. Kim, J. Am. Chem. Soc. 2013, 135, 17969-17977. 
[30] a) R. W. Sinkeldam, A. J. Wheat, H. Boyaci, Y. Tor, Chemphyschem 2011 12, 567-570; b) R. W. Sinkeldam, P. Marcus, D. Uchenik, Y. Tor, Chemphyschem 2011, 12, 2260-2265; c) P. A. Hopkins, R. W. Sinkeldam Y. Tor, Org. Lett. 2014, 16, 5290-5293; d) A. A. Tanpure, S. G. Srivatsan, Chembiochem 2012, 13, 2392-2399; e) A. A. Tanpure, S. G. Srivatsan, Chembiochem 2014, 15, 1309-1316; f) J. Riedl, R. Pohl, N. P. Ernsting, P. Orság, M. Fojta, M. Hocek, Chem. Sci. 2012, 3, 2797-2806; g) J. Riedl, P. Ménová, R. Pohl, P. Orság, M. Fojta, M. Hocek, J. Org. Chem. 2012, 77 8287-8293; h) Y. Saito, A. Suzuki, Y. Okada, Y. Yamasaka, N. Nemoto, I. Saito, Chem. Commun. 2013, 49, 5684-5686.

[31] a) R. W. Sinkeldam, P. a Hopkins, Y. Tor, Chemphyschem 2012, 13, 3350-3356; b) J. Riedl, R. Pohl, L. Rulíšek, M. Hocek, J. Org. Chem. 2012, 77, 1026-1044.

[32] A. P. Demchenko, J. Fluoresc. 2010, 20, 1099-1128.
[33] W. Melhuish, J. Phys. Chem. 1961, 97, 229-235.

[34] a) A. D. Becke, J. Chem. Phys., 1993, 98, 5648-5652; b) C. T. Lee, W. T. Yang and R. G. Parr, Phys. Rev. B: Condens. Matter, 1988, 37, 785-789; C) P. J. Stephens, F. J. Devlin, C. F. Chabalowski M. J. Frisch, J. Phys. Chem., 1994, 98, 11623-11627.

[35] M. J. Frisch, GAUSSIAN 09, Revision D.01, Gaussian, Inc., Wallington Ford, CT, 2009

[36] a) K. Burke, J. Werschnik, E. K. U. Gross, J. Chem. Phys., 2005, 123 62206-62209; b) M. A. L. Marques and E. K. U. Gross, Annu. Rev. Phys. Chem., 2004, 55, 427-455.

[37] a) V. Barone, M. Cossi, J. Phys. Chem. A, 1998, 102, 1995-2001; b) A. Klamt, G. Schuurmann, J. Chem. Soc., Perkin Trans. 2, 1993, 799-805.

\section{FULL PAPER}

2-aminopurine has for long been the standard for fluorescent base analogues. Here we present four fluorescent probes suitable for the replacement of adenine in nucleic acids. Based on their high structural similarity to their parent compound qA they have the potential to be excellent A-analogues as well. Their improved photophysical properties also suggests that they could be significantly brighter than 2aminopurine inside nucleic acid systems.

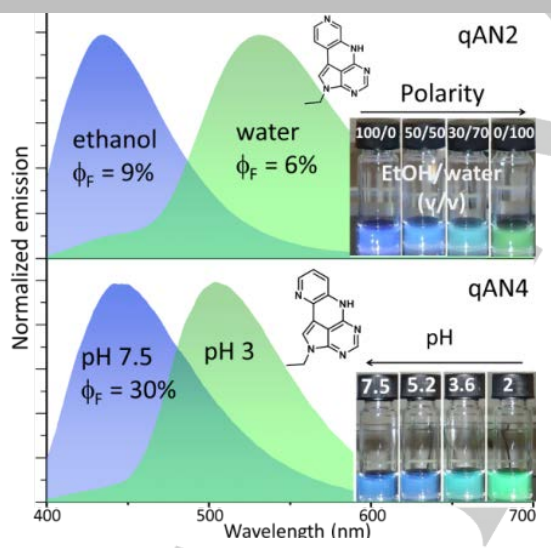

Blaise Dumat, Mattias Bood, Moa S. Wranne Christopher P. Lawson, Anders Foller Larsen, Søren Preus, Jens Streling, Henrik Gradén, Eric Wellner, Morten Grøtli* and L. Marcus Wilhelmsson*

Page No. - Page No.

Second-generation fluorescent quadracyclic adenine analogues: Environment responsive probes with enhanced brightness 